Gastroenterologe 2011 · 6:179-180

DOI 10.1007/s11377-011-0555-8

Online publiziert: 14. April 2011

(c) Springer-Verlag 2011

H. Seidl ${ }^{1}$ M. Fried ${ }^{2} \cdot$ W. Schepp ${ }^{1}$

${ }^{1}$ Klinik für Gastroenterologie, Hepatologie und gastroenterologische Onkologie, Städtisches Klinikum München GmbH, Klinikum Bogenhausen, München

${ }^{2}$ Klinik für Gastroenterologie und Hepatologie, Universitätsspital Zürich

\title{
Funktionsdiagnostik - State of the Art 2011
}

\section{Vom Syndrom zur pathophysiologisch basierten Differenzialdiagnose}

Als Lokomotive der gastroenterologischen, klinisch orientierten Forschung stand in den letzten Jahrzehnten die Endoskopie im Vordergrund. Meilensteine wie die flexible Endoskopie, Chip-on-the-TipTechnologie, ausgefeilte (immun-)histologische Biopsieaufarbeitung und vielfältige therapeutische Innovationen hielten diese in Fahrt. Dennoch machen Patienten mit funktionellen Beschwerden, die mit unauffälligem endoskopischem Befund durch das verfügbare Raster fallen, bis $\mathrm{zu} 40 \%$ einer gastroenterologischen Praxis aus. Hinzu kommt, dass nur ein Fünftel der Patienten mit funktionellen Beschwerden einen Arzt aufsucht [1].

\section{(2) Die Vielfalt der therapeutischen Optionen erfordert eine differenzierte Diagnose- und Indikationsstellung}

In einer symptomorientierten Herangehensweise - gemäß der historischen Weisheit „Alle Wege führen nach Rom“ wurden häufig klinische Fragenkataloge zur Diagnosestellung funktioneller Erkrankungen herangezogen. Zuletzt wurden in der 3. Revision der Rom-Kriterien (2006) funktionelle Krankheitsbilder anhand von Symptomengruppen eingeordnet. Aber auch dieser pragmatische Ansatz hat sich klinisch als wenig alltagstauglich erwiesen. In Studien fand sich eine schwache Diskriminationskraft, mit bis zu 65\% Überlappung zwischen den so definierten Krankheitsgruppen. Die Hälf- te der Patienten zeigte binnen Jahresfrist einen Wechsel der symptombasierten $\mathrm{Zu}$ ordnung [2]. Aktuelle Leitlinien, wie die Novelle der S3-Leitlinie der DGVS zum Reizdarmsyndrom, entfernen sich daher wieder von zu engen Symptomkriterien.

Bedeutende neue Einsichten in Physiologie und Pathophysiologie des komplexen digestiven Apparates führten vom früher üblichen syndromalen Ansatz zur heutigen pathophysiologischen Klassifikation und Therapie: Hierzu zählen Neurotransmitter und Steuermechanismen des enterischen Nervensystems, interstitielle Cajal-Zellen als intestinaler Schrittmacher und neuromuskulärer Mediator, immunhistochemische Darstellung von spezifischen Erkrankungen der integralen neuromuskulären Komponenten, typische neuro- und myopathische Motilitätsphänomene - um nur einige zu nennen. Die folgenden Beiträge beleuchten innovative, moderne Verfahren (wie die intraluminale Impedanzmessung oder die High-Resolution-Manometrie), die Einblicke in die Pathophysiologie erlauben und zunehmende therapeutische Relevanz haben.

Der praktischen Anwendung solch neuer Erkenntnisse und Untersuchungsmethoden steht oft das Vorurteil einer fehlenden klinischen Konsequenz entgegen. Zu Unrecht, hält man sich die neuesten therapeutischen Fortschritte vor $\mathrm{Au}$ gen. Beispielsweise sind mit Lubiproston oder Prucaloprid wieder verträgliche Prokinetika verfügbar. Die Neuromodulation durch sakrale oder intestinale/gastrale Schrittmacher hat den Sprung in die klinische Anwendung geschafft. Dank jahrzehntelanger Erfahrungen wurden chirurgische Techniken optimiert, wie z. B. die laparoskopische modifizierte Heller-Myotomie bei Achalasie.

Aber gerade diese Vielfalt therapeutischer Optionen setzt eine differenzierte Diagnose- und Indikationsstellung voraus. In der Grenzfindung zwischen konservativen und operativen Verfahren erlaubt eine zeitgemäße Funktionsdiagnostik die Abschätzung von Risiken und Prognose und damit ein integriertes interdisziplinäres Vorgehen.

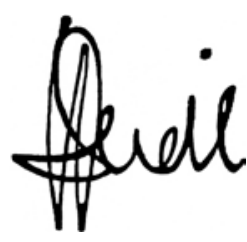

Holger Seidl

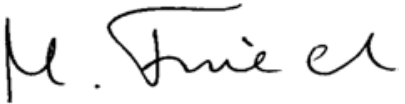

Michael Fried

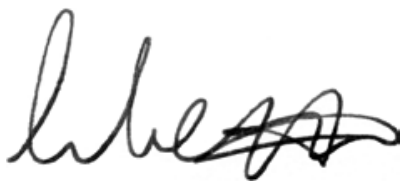

Wolfgang Schepp 


\section{Korrespondenzadressen}

\section{Dr. H. Seidl}

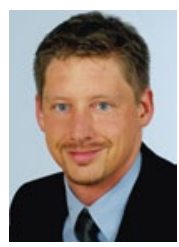

Klinik für Gastroenterologie, Hepatologie und gastroenterologische Onkologie, Städtisches Klinikum München $\mathrm{GmbH}$, Klinikum Bogenhausen Englschalkinger Str. 77, 81925 München Holger.Seidl@gmx.com

\section{Prof. Dr. M. Fried}

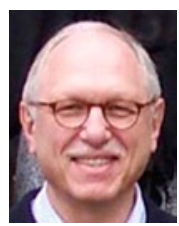

Klinik für Gastroenterologie und Hepatologie, Universitätsspital Zürich 8091 Zürich

Schweiz

Michael.Fried@usz.ch

\section{Prof. Dr. W. Schepp}

Klinik für Gastroenterologie,
Hepatologie und
gastroenterologische
Onkologie, Städtisches
Klinikum München GmbH,
Klinikum Bogenhausen
Englschalkinger Str. 77,
81925 München
schepp.wolfgang@t-online.de

\section{Literatur}

1. Talley NJ, Silverstein MD, Agréus L et al (1998) AGA technical review: evaluation of dyspepsia. American Gastroenterological Association. Gastroenterology 114: 582-595

2. Agréus L; Svärdsudd K; Nyrén O et al (1995) Irritable bowel syndrome and dyspepsia in the general population: overlap and lack of stability over time. Gastroenterology 109: 671-680

\section{Themenübersicht}

„Der Gastroenterologe“ bietet Ihnen umfassende und aktuelle Beiträge zu interessanten Themenschwerpunkten aus allen Bereichen der Gastroenterologie und Hepatologie.

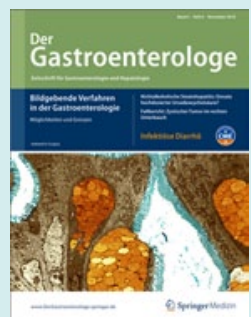

\section{Schwerpunktthemen 2010}

- Heft 1/10 Hereditäre gastroenterologische Erkrankungen

- Heft 2/10 NASH \& Co.

- Heft 3/10 Interdisziplinarität in der Gastroenterologie

- Heft 4/10 Proktologie

- Heft 5/10 Gastrointestinale Onkologie

- Heft 6/10 Bildgebende Verfahren in der Gastroenterologie

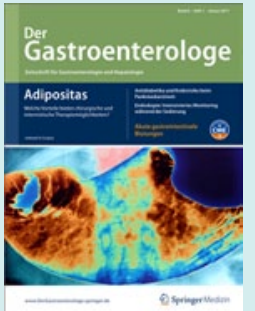

\section{Schwerpunktthemen 2011}

- Heft 1/11 Adipositas

- Heft 2/11 Molekulargenetische Analysen

- Heft 3/11 Funktionsdiagnostik

- Heft 4/11 GI-Trakt und Nachbarorgane

- Heft 5/11 Palliativmedizin in der Gastroenterologie

- Heft 6/11 Kinder- und Jugendgastroenterologie

\section{Volltextarchiv „Der Gastroenterologe“}

Abonnenten haben online Zugriff auf alle Beiträge im elektronischen Volltextarchiv unter www.DerGastroenterologe.springer.de

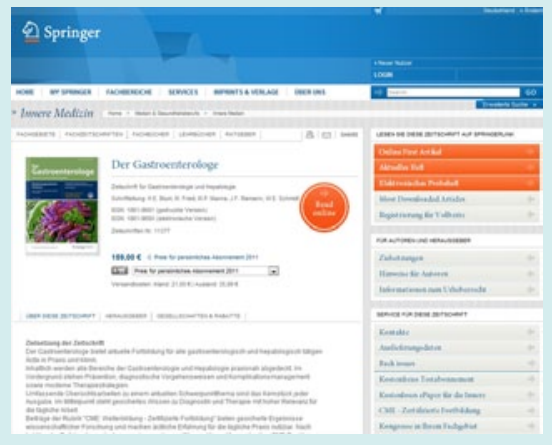

Haben Sie noch Fragen oder Anmerkungen? Wir freuen uns über Ihre E-Mail an: annette.gasser@springer.com

Ihre Redaktion

Fachzeitschriften Medizin/Psychologie 